\title{
THE TOPOLOGICAL FIXED POINT PROPERTY AN ELEMENTARY CONTINUUM-THEORETIC APPROACH
}

\author{
ROMAN MAŃKA \\ Institute of Mathematics, Polish Academy of Sciences \\ Śniadeckich 8, P.O.B. 21, 00-956 Warsaw \\ E-mail: manka@impan.gov.pl
}

\begin{abstract}
A set contained in a topological space has the topological fixed point property if every continuous mapping of the set into itself leaves some point fixed. In 1969, R. H. Bing published his article The Elusive Fixed Point Property, posing twelve intriguing and difficult problems, which exerted a great influence on the study of the fixed point property. We now present a survey article intended for a broad audience that reports on this area of fixed point theory. The exposition is also intended to give an introduction to the current study of the fixed point property from the viewpoint of an elementary continuum theory.

This article is an expanded version of an invited lecture given by the author at the International Conference on Fixed Point Theory and its Applications in Memory of Jim Dugundji (1919-1985), Będlewo, Poland, August 1st to 5th, 2005.

The author wishes to thank Kazuhiro Kawamura and Mirosław Sobolewski for several suggestions that led to the improvement of this article. The author's special thanks are due to Charles L. Hagopian for his careful reading the penultimate typescript and supplying valuable remarks and comments that corrected the article. Special thanks are also due to the referee for valuable suggestions.
\end{abstract}

1. Introduction. A set contained in a topological $\left(T_{1}\right)$ space has the fixed point property, briefly FPP, if every continuous mapping $f$ of the set into itself has a fixed point, i.e. there is a point $p$ of the set which satisfies the identity $p=f(p)$. The FPP is a topological property, i.e., if a set has the FPP, then its homeomorphic image has the FPP. Arcs, i.e. homeomorphic images of a closed interval of the real numbers, are the simplest sets which

2000 Mathematics Subject Classification: Primary 54H25; Secondary 54F15.

Key words and phrases: contractible sets, uniquely arcwise connected sets (uac sets), tree-like continua.

The paper is in final form and no version of it will be published elsewhere. 
have the FPP. Simple closed curves, i.e. homeomorphic images of a circle, are the simplest connected sets which do not have the FPP. Disks, i.e. homeomorphic images of a closed ball of the plane, have the FPP, but this requires a non-trivial proof (cf. Shashkin 1989 for the elementary approach).

Which sets have the fixed point property FPP? Can we describe them? Such questions arise at the beginning of the study of the FPP. We will survey their present state in relation to the widely known article by R. H. Bing 1969. To this end, some elementary topological notions will be needed. Instead of "a set contained in a topological $\left(T_{1}\right)$ space" we will write "a set in a space".

A set in a space is a continuum if it is connected and compact. A set in a space is arcwise connected provided that every pair of its points constitutes end points of an arc contained in this set. It is a classical result of the point set topology that every continuous image of an arc is arcwise connected, and so every continuous image of an arcwise connected set is arcwise connected (continuous images of an arc have different names in the literature: Peano (or continuous) curves, Peano continua, locally connected continua, or paths, the last term being used especially in the homotopy theory where the pathwise connectivity is also used instead of the arcwise connectivity). We will use the term locally connected continuum for any set which is a continuous image of an arc, because this is equivalent to saying that it is a (metrizable) continuum every point of which has an arbitrarily small connected neighborhood. The locally connected continua are arcwise connected whenever they are metrizable. Further, (non-empty) metrizable continua will be called shortly continua.

Three topological notions will play a key role in what follows: contractible sets, uniquely arcwise connected sets, and tree-like continua.

A set in a space is contractible if there is a homotopy joining the identity mapping with a constant mapping in the set. Convex sets (in a topological vector space) and AR's as well are contractible and locally contractible (cf. Borsuk 1967, p. 26 and 28, and 101). Of course, all (topological) cones are contractible sets. Every contractible set is simply connected, i.e. it is arcwise connected (三0-connected) and 1-connected; in other words, its fundamental group is trivial (cf. e.g. Borsuk 1967, p. 30 and 50).

A set in a space is uniquely arcwise connected, shortly uac, if it is arcwise connected and contains no simple closed curve, i.e. every two of its points are the end points of a unique arc lying in the set between these points. By a classical result of dimension theory, a one-dimensional arcwise connected continuum with the FPP must be uac. Of course, every uac set is simply connected.

A set in a space is a tree-like continuum if it is the limit of an inverse sequence of trees, i.e. one-dimensional acyclic polyhedra (all polyhedra mentioned here will be finite and connected). This is equivalent to saying that it is a continuum such that for each $\varepsilon>0$ there is a tree and a continuous mapping of the continuum onto the tree all point-inverses of which have diameters less than $\varepsilon$. If all trees in the above definitions are reduced to arcs, then the resulting continuum is called an arc-like continuum (or a snake-like continuum, or a chainable continuum). There are also characterizations of tree-like and arc-like continua which use a covering technique, and other techniques as 
well. It is a classical result (of Bing 1951 - in one direction) that a subset of the plane is a tree-like continuum if and only if it is a one-dimensional non-separating plane continuum, i.e. its complement to the whole plane is connected. Arc-like continua are always planar, i.e. possess homeomorphic images contained in the plane $E^{2}$, as proved by Bing 1951 . Tree-like continua need not be planar but they always have a homeomorphic image in the space $E^{3}$, by some classical results of dimension theory. Let us note that the treelike continua which are locally connected coincide with dendrites, i.e. locally connected continua containing no simple closed curve. One should also realize that every continuum of dimension not exceeding a non-negative integer $n$ is the limit of an inverse sequence of polyhedra of dimension not exceeding the number $n$ (by classical topological results).

Of course, tree-like continua need not be arcwise connected. This fact has considerable, and very difficult, consequences as will be seen in Chapter 4 of this survey. On the other hand, tree-like continua which are arcwise connected lie in a center of the above described three classes of sets: contractible, uac, and tree-like - when they are considered with respect to the topological fixed point property FPP (as we will see in what follows).

Instead of giving a formulation of a result we will often mark it only with the name of the author and the year of the publication. Because, most frequently, the title of the quoted paper stands for the formulation and there is no need to repeat it in the present paper. This is the reason for including the references to the main body of the paper, in a separate chapter with distinguishing the surveys about the fixed point property that appeared after Bing's expository article 1969.

2. The fixed point property before Bing's article 1969. If a set is not connected then it obviously does not have the topological fixed point property FPP. Only a little can be said on the FPP for non-compact connected sets (but cf. e.g. (7) and (19) below, Lelek 1972, Mańka 1978b, Problem $3^{0}$, p. 434 (cf. Marsh 1987) and Problem $4^{0}$, p. 435 (cf. Jones 1988), Okhezin 1985 and 1995, Tominaga 1992, Gutek 1988, Dijkstra 2006, and Niemiec 2006). Therefore the FPP is studied mainly for continua.

A long line of the study concerning Brouwer fixed point theorem and the FPP for compact convex sets includes: Brouwer 1909, 1912, KKM 1929, Schauder 1930, Tychonoff 1935, Kakutani 1943, Dugundji 1951, Klee 1955, . . ; cf. Mawhin 2005 (preprint) and also Papini 1993. Another theory concerns polyhedra - cf. the survey by R. F. Brown 1999. But, as we have indicated, a natural domain of the study of the FPP consists of arbitrary continua.

An extensive survey of results on the FPP was contained in a tract by van der Walt 1963. It contained almost everything known at that time about the FPP.

In 1969, R. H. Bing published an expository article The elusive fixed point property which revealed in a surprisingly simple manner many essential gaps in that knowledge and exerted a great influence on the further study of the topological fixed point property FPP. We recall what was known about the FPP for continua when Bing published the article.

(1) Scherrer 1926 proved that dendrites have the FPP for homeomorphisms, and this was generalized in Nöbeling 1932 that all dendrites have the fixed point property; 
(2) Borsuk 1931 introduced a class of AR's, which have the FPP. Also the products of sequences of AR's of AR's are AR's, and if the common part of two AR's is an AR then their union is an AR as proved by Aronszajn and Borsuk 1932;

(3) Borsuk 1932 proved that a locally connected plane continuum has the FPP if and only if it does not separate the plane, or equivalently it is an AR (the notion of cyclic elements of Whyburn was used (cf. also Ayres 1930, p. 335, Note from the Editors), in particular, it was there proved that a continuum is a dendrite if and only if it is a one-dimensional AR);

(4) Borsuk 1935 constructed a 3-dimensional locally connected continuum in $E^{3}$ which does not separate the space $E^{3}$ and does not have the FPP, even for homeomorphismsproving that the FPP is not a homological property; Borsuk's example is the intersection of a decreasing sequence of balls of $E^{3}$;

(5) Hamilton 1938 revealed a connection between the FPP and the notion of the decomposability of continua. A continuum is decomposable if it is the union of its two proper subcontinua. He established the FPP for homeomorphisms of the non-separating plane continua which are hereditarily decomposable, i.e. all non-degenerated subcontinua are decomposable. Also, he established the fixed point property for homeomorphisms for the non-separating plane closures of simply connected open sets with a hereditarily decomposable boundary;

(6) Verchenko 1940 constructed a two-dimensional locally connected continuum lying in $E^{3}$ which does not separate $E^{3}$ and does not have the FPP. Also he constructed a 3 -dimensional continuum lying in the Euclidean space $E^{4}$ which has analogous properties of the example of Borsuk 1935 and in addition is simply connected;

(7) Gail S. Young 1946 established the FPP for uac $\left(T_{2}\right)$ spaces in which the union of every increasing sequence of arcs is contained in an arc - in particular, the cone over any set containing no arc, and the lexicographical product of the set of all countable ordinals with a half-open interval of the reals both have the FPP (with respect to the interval topology);

(8) Hamilton 1951 proved that each arc-like continuum has the FPP;

(9) Kinoshita 1953 constructed a contractible two-dimensional continuum in $E^{3}$ without the FPP and this implies the cone over this continuum also does not have the FPP;

(10) Borsuk 1954 proved that contractible (even Vietoris-acyclic and arcwise connected) one-dimensional continua (三 curves) have the FPP. The proof was based on interpreting the Vietoris acyclicity for arcwise connected curves in terms of point set topology. He proved that the homological acyclicity is equivalent to the hereditary unicoherence. Recall that a continuum is hereditarily unicoherent if all its subcontinua are unicoherent, i.e. if the subcontinuum is the union of two subcontinua, then the intersection of these continua is connected. Arcwise connected hereditarily unicoherent continua were distinguished as separate object of study under the name dendroids by Knaster 1959. Of course, every dendrite is a dendroid and every dendroid is a uac curve. Hereditarily unicoherent and hereditarily decomposable continua were called $\lambda$-dendroids by Knaster 1960, and every dendroid is a $\lambda$-dendroid by a lemma of Borsuk 1954. Let us add here that shortly after 
the article by Bing 1969, Cook 1970 proved that dendroids and $\lambda$-dendroids as well are tree-like continua, and thus the tree-like continua which are arcwise connected coincide with dendroids;

(11) Dyer 1956 and Lokutsievskiu 1957 established the FPP for products of arbitrarily many arc-like continua (by methods quite different from each other);

(12) Gail S. Young 1960 gave an example of a uac curve without the FPP (vaguely presented - necessary details was given by Mańka 1994, but the first correctly described example of a uac curve without the FPP was given by Sobolewski 1986);

(13) L. E. Ward, Jr. 1961 proved that an arcwise connected continuum has the FPP for continuum-valued upper semicontinuous mappings if and only if it is a dendroid;

(14) Klee 1961 introduced a notion of the proximate fixed point property and proved that it is invariant under homeomorphisms and retractions as well. He also prove that the proximate fixed point property is stronger than the FPP. The property was also introduced by Sobolewski 1988, under another definition which was proved by Sanjurjo 1989 to be equivalent with the definition of Klee 1961; cf. Čerin and Sanjurjo 1996 for a present state of the study of this property. We would like to ask here whether dendroids have the proximate fixed point property;

(15) Bing 1967 constructed a two-dimensional locally connected continuum in $E^{3}$ which is the intersection of a decreasing sequence of balls of $E^{3}$ and does not have the FPP for homeomorphisms of the continuum onto itself;

(16) Knill 1967 proved that the cone over a contractible continuum has the fixed point property if and only if its cylinder has the FPP, and constructed a two-dimensional contractible continuum $B$ in $E^{3}$ with the FPP the cone over which does not have the FPP - the contractible continuum $B$ has the property that there is a 3-cell $A$ (三 a homeomorphic image of a ball of $E^{3}$ ) such that $A \cap B$ is a disc, and $A \cup B$ does not have the FPP. He proved also that the cone over a circle with a spiral limiting on the circle does not have the FPP, and posed the problem whether the cones over tree-like continua have the FPP. A negative and stronger solution of the problem for plane $\lambda$-dendroids was recently announced by Illanes 2005 (preprint);

(17) Lopez 1967 constructed a 17-dimensional polyhedron $P$ such that $P$ has the FPP and the cylinder $P \times[0,1]$ over $P$ does not have the FPP, and that

$(17)^{*}$ There is a disk $D$, with $D \cap P$ being an arc, such that $P \cup D$ does not have the FPP (in particular, this answered in the negative a problem raised by L. E. Ward, Jr. 1966).

(18) Bell 1967 and Sieklucki 1967, 1968 proved that for every continuous mapping of a non-separating plane continuum into itself there is an invariant indecomposable continuum (三 continuum which is not decomposable) contained in its boundary; in particular, if the boundary of non-separating plane continuum is hereditarily decomposable then it has the FPP;

(19) Holsztyński 1969 gave a detailed study, and generalizations of the ideas of (7) and $(10)$ to $\left(T_{1}\right)$ spaces - in particular he gave another, game theoretical, proof of the main results of $(7)$ and $(10)$; 
(20) Bing 1969 gave a (non-planar) uac curve $P$ which has the FPP and the above property $(17)^{*}$. Also he gave two singular two-dimensional continua in $E^{3}$ without the FPP, complementing the ones which had been given previously in (6), (9), (15), (16).

Despite of the positive results (1), (2), (3), (5), (7), (8), (10), (11), (13), (18) and (19), one could say the FPP is "elusive", which means "difficult to catch" (cf. the Longman Dictionary of Contemporary English, 1987). Simply, the FPP is difficult to catch because no known topological notion of acyclicity (in algebraic topology, in particular) describes the continua which have the FPP. To illustrate this, we point out that only two of the above results are of the desired kind: (3) and (13) (the most significant result of this kind has been given quite recently, by Hagopian 1996; it will be presented in discussing Question 4 in Chapter 4). But, what about other classes of continua and mappings - we have only counterexamples or open problems!?! In fact, Bing 1969 asked twelve provocative, simple, and yet difficult, questions concerning the FPP. Eight of them have been answered.

3. The problems of Bing 1969 and their solvers. As we have said, Bing named his problems as question. We give now the full list of the problems, literally, as they appear in the Bing's article. After their formulations, we present their status and solvers.

QUESTION 1. Is there a two dimensional polyhedron with the fixed point property which has even Euler characteristic?

Open problem.

Question 2. Does each tree-like continuum have the fixed point property?

No: D. P. Bellamy 1978, 1980.

Question 3. Does the intersection of each decreasing sequence of disks have the fixed point property?

Open problem.

QUESTION 4. Does each arcwise connected compact planar continuum containing no simple closed curve have the fixed point property?

Yes: C. L. Hagopian 1978, 1979.

Question 5. Does $X \times[0,1]$ have the fixed point property?

The above $X$ represents the Bing's uac curve, an example in his article 1969.

Yes: W. L. Young 1970.

QUESTION 6. If $C$ is an arcwise connected continuum which contains no simple closed curve, does each homeomorphism of $C$ into itself leave some point fixed?

Yes: L. Mohler 1975.

QUESTION 7. If $C$ is a plane continuum with the fixed point property and $D$ is a disk that intersects $C$ in an arc, must $C \cup D$ have the fixed point property?

No: C. L. Hagopian and J. R. Prajs 2005. 
QUESTION 8. What is the lowest dimension for such a polyhedron $P$ as in $(17)^{*}$ ?

Open problem.

QUESTION 9. If a compact one-dimensional continuum has the fixed point property, does its cartesian product with a segment?

No: M. Sobolewski 2005.

QUESTION 10. If a bounded plane continuum has the fixed point property, does its cartesian products with an arc?

Open problem.

Question 11. If $P$ and $Q$ are polyhedra without local separating points but with the fixed point property, must $P \times Q$ have the fixed point property?

No: G. E. Bredon 1971 (see Fadell 1971 for a reformulation of Bredon's solution).

Question 12. Does each homeomorphism of a contractible continuum onto itself leave some point fixed?

No: J. M. Łysko 1972.

4. Results following the problems of Bing 1969. We leave Problems 1,8 and 11 concerning polyhedra out of further comments (some contribution can be deduced from the fixed point theory for polyhedra; cf. e.g. the surveys by R. F. Brown 1982, 1999).

The FPP is sometimes considered not only for continuous mappings but also for some other classes of mappings, e.g. homeomorphisms, deformations (三 the continuous mappings which are homotopic to the identity mapping), continuum-valued upper semicontinuous mappings, etc., and we will write then $\operatorname{FPP}($ hom $), \operatorname{FPP}($ def $), \operatorname{FPP}(c$-func), etc., respectively.

QUESTION 2. Does each tree-like continuum have the FPP?

Cook 1970 proved that dendroids and $\lambda$-dendroids are tree-like continua. Thus the result of Borsuk 1954, and the first result of Hamilton 1938 as well, appeared to be partial answers to this question. Mańka 1975, 1976 proved that every hereditarily decomposable tree-like continuum ( $\equiv \lambda$-dendroid by Cook 1970) has the $\operatorname{FPP}(c$-func); in particular, it has the FPP which answered a question of Knaster 1960 in the positive. Gurevich 1978 showed that the proof of Mańka 1976 is valid for so called non-metric $\lambda$-dendroids by reformulating this proof in terms of lattice theory (cf. Mańka 1978a). Mańka 2005 (preprint) gave a common generalization of the FPP for dendroids and the lattice-theoretical fixed point theorem of Tarski 1949, 1955, by using an idea of Knaster 1928. This shows that there is a great diversity of methods which may apply to Question 2 (cf. also Mańka 1987c). Hagopian 1998 proved the FPP(def), even the FPP for arc-component preserving continuous mappings of tree-like continua. Other positive contribution to Question 2 were given by Fugate and McLean 1981, and Oversteegen and Tymchatyn 1981-both involving periodic homemorphisms, and Marsh 1984 and 1986-specializing the inverse sequences defining tree-like continua. 
Fugate and Mohler 1977 proved that for every tree-like continuum without the FPP there is a tree-like continuum without the FPP(hom). After the solution of Bellamy 1980, several related examples were given by Oversteegen and Rogers 1980 and 1982, Fearnley and Wright 1993, and Minc 1990, 1992, 1996, 1999, 2000. For instance, Minc 2000 gave an example of a tree-like continuum without the FPP which is hereditarily indecomposable, i.e. all of its subcontinua are indecomposable (a brief survey on indecomposable continua was given by Kennedy 1995). Minc 1999 gave an example of a tree-like continuum which does not have the FPP and is weakly chainable, named after being continuous images of the pseudoarc of Knaster 1922 - a unique (up to homemorphisms) hereditarily indecomposable arc-like continuum (cf. the surveys by Kennedy 1995 and Lewis 1999).

\section{Question 3. Does the intersection of each decreasing sequences of disks have the FPP?}

The question represents indeed one of the most difficult problems in mathematics (cf. Bing 1967): does each non-separating plane continuum $X$ have the FPP? Some commentary on this question was given by Bing 1981. In addition to it, we mention that Iliadis 1970 and Minc 1972 gave some approaches to the Bell-Sieklucki theorem which are different from the original ones (and different from each other). From the theorem of Bell and Sieklucki, Hagopian 1971 derived the FPP for every arcwise connected nonseparating plane continuum $X$. Hagopian 1972 and independently Krasinkiewicz 1973 extended this result to every such $X$ that is $\lambda$-connected (we do not explain the notion here, but note merely that the planar $X$ is $\lambda$-connected if and only if every pair of its point lies in a hereditarily decomposable subcontinuum of $X$ ). Minc 1990 established the FPP for the $X$ all indecomposable subcontinua of the boundary of which are contained in a weakly chainable subcontinuum of $X$; in particular, each non-separating weakly chainable plane continuum has the FPP. Hagopian 1998 established the FPP(def) for every non-separating plane continuum $X$. Minc 1976, 1980 showed that there are continua in $E^{3}$ which are locally connected and separate $E^{3}$, but they have the FPP. Thus, in view of $(3),(4),(6),(15)$ and (20), there is a great difference between the study of the FPP in $E^{2}$, and in $E^{3}$.

Let us note that Question 3 is open even in the class of (one-dimensional indecomposable) non-separating plane continua all proper subcontinua of which are arcs (a question of Hagopian 1991); it is not even known whether such continua have the FPP(hom) - also in the case when the continua are inverse limits of simple triods, i.e. unions of three arcs emanating from a common end point, disjoint outside the end point.

Some other contributions to Questions 2 and 3, and some other related questions, are given by Hagopian 1991. The approach of Sieklucki 1974, 1975, 1980 to above Questions 2 and 3 restricted to the dimension 1 still looks promising. One could expect also a development of the idea of Thompson 1971, Sieklucki 1971, Minc 1972, Iliadis 1983, Bell 1983 and Garrett 2004.

Remark on pursuit method. Let us add that not only the result of Borsuk 1954 (cf. (10)) but also his proof initiated a new approach to the FPP. This proof originated a general pursuit method for proving positive results on the FPP. Bing 1969 considered it as the dead-end method, and Hagopian 1986 extended it to the more dynamical dog-chases- 
principle. The pursuit method has been applied under similar forms in many papers, e.g. by Ward 1961, Holsztyński 1969, W. L. Young 1971, Mohler 1975, Hagopian 1978-1998, Mańka 1987(a-c), Bellamy 1994, Fugate and Mohler 1995, and, in some doubly abstracted setting by Mańka 1976, 2002. Some fundamentals of the general pursuit method are a subject of the paper by Mańka 2005.

Question 4. Does each uac plane continuum have the FPP?

After his solution to this problem in 1978, 1979, Hagopian 1996 gave a definitive result on the FPP for the plane continua which are arcwise connected - they have the FPP if and only if they are simply connected.

Question 5. If $P$ is the Bing's uac curve, does $P \times[0,1]$ have the FPP.

The original Bing's 1969 example $P$ (non-planar) was merely sketched, and the proof of its properties given by him was not complete. This was defined and proved in a more general setting by Mańka 1994. We expect that the solution of Question 5 by W. L. Young 1970 can be extended to the generalized setting.

Question 6. If $C$ is an uac continuum, does $C$ has the $\operatorname{FPP}($ hom $)$ ?

Mohler and Oversteegen 1985 extended the solution of Mohler 1975 to the locally oneto-one continuous mappings, and Fugate and Mohler 1995 - to arc-preserving continuous mappings. Hagopian 1986 established the $\operatorname{FPP}($ def $)$ for every uac continuum. To the examples discussed in (12), let us add the example of Holsztyński 1969 (cf. Hagopian and Mańka 2003). Sobolewski 2004 (preprint) constructed a weakly chainable uac curve without the FPP, answering a question of Bellamy 1994.

Question 7. Is there a plane continuum $C$ with the FPP and property $(17)^{*}$ with $P=C$ ?

The plane curve of Hagopian and Prajs 2005 is hereditarily decomposable and even rational, i.e. it has a base of neighborhoods with countable boundaries. If $C$ and $D$ are any curves with the FPP and $C \cap D$ is a dendrite, then $C \cup D$ has the FPP as proved by Shtanko 1964. Mańka 1987a and 1990 proved that nothing more general can be used in place of the dendrite in Shtanko's theorem, e.g. the assumption that $C \cap D$ is a dendrite cannot be replaced even by the statement that $C \cap D$ is a hereditarily contractible continuum. However if the union $C \cup D$ of the curves $C$ and $D$ with the FPP lies on the plane $E^{2}$ then we do not know if $C \cup D$ has the FPP whenever the common part $C \cap D$ is arcwise connected (and what about the case when the common part $C \cap D$ is a dendroid); cf. the end of the paper by Mańka 1990.

QUESTION 9. If a curve has the FPP, does its cartesian product with a segment?

Sobolewski's 2005 counterexample (which contains a solenoid) is complemented by a theorem of Mańka 2002 which answers Question 9 in the positive for $\lambda$-dendroids; moreover it is stated in this paper that any counterexample to this question must contain a one-to-one continuous image of a half-line that is not contained in an arc, which can be derived from a theorem of Okhezin 1985. Sobolewski 2005 asked if Question 9 has a positive answer when the curve is also assumed to be hereditarily decomposable. 
There are also several important results and problems concerning the FPP for products of curves, cones and hyperspaces, see e.g. Fugate 1971, and Eberhart and Fugate 1971, Krasinkiewicz 1974, Hagopian 1975, Tominaga 1984 and 1992, Nadler 1978, and Illanes and Nadler 1999. Let us add that spirals are irreducible continua, i.e. they contain two points such that no other subcontinuum of them contains both of these points. By some classical topological results, each rational irreducible continuum admits a unique upper semicontinuous decomposition into nowhere dense subcontinua, called tranches or layers, with the property that its hyperspace is an arc. Hagopian 2003 (cf. also Hagopian and Mańka 2005) constructed irreducible rational continua without the FPP all tranches of which have the FPP, answering an old problem of Gordh (cf. Problem 43, p. 371, in Lewis 1983).

QUESTION 10. If a plane continuum has the FPP, does its cartesian product with an arc?

The answer is yes for locally connected non-separating plane continua because they are AR's by a theorem of Borsuk 1932. The same is true for all hereditarily decomposable non-separating plane continua by a theorem of Mańka 2001. One could ask whether there is a common counterexample to the above Questions 7, 9 and 10.

Question 12. Does each contractible continuum have the FPP(hom)?

The lack of the FPP for two-dimensional contractible continua of Kinoshita 1953, Knill 1967, and Bing 1969 was explained by Sieklucki 1985 who added to the solution of Łysko 1972 the following question: Does each cone (or even generalized cone by Sieklucki 1985) over a compact set have the $\mathrm{FPP}($ hom $)$ ? We add here the following question: Does each two-dimensional contractible continuum lying in $E^{3}$ have the $\operatorname{FPP}($ hom $)$ ? This is a special case of a question raised by Łysko and the author, in a conversation some years ago: Does each two-dimensional contractible continuum have the $\operatorname{FPP}($ hom)?

Although there are some open problems on the topological fixed point property FPP for continua of arbitrary dimension (for instance, whether contractible and locally contractible continua have the FPP, or, what are the limits of definability of the Lefschetz number non-vanishing for all continuous mappings of continua - a significant approach to these problems has appeared in Cauty 2005 when the present article was prepared to print), the above presented works on the problems of Bing 1969 show that the study of the FPP is concentrated on low dimensional continua, i.e. lying in the space $E^{3}$, and special difficulties can be expected in the ones which have the lowest dimension 1 . In this scope, the fundamental question stated at the beginning of the present paper, can be more specifically stated as follows: Which contractible continua have the FPP or FPP(hom)? Which uac continua have the FPP? Which tree-like continua have the FPP? What about the products of one-dimensional continua, and the cones and hyperspaces?

In particular, the question (of Bellamy 1995): whether planar tree-like continua have the FPP, consists the one-dimensional part of the classical old problem: does each nonseparating plane continuum have the topological fixed point property FPP? FPP(hom)? For some contribution to the last question, formulated by Hagopian 1991, the readers are referred to Bell 1976, 1978 and Krystyna Kuperberg 1989, 1991. 
5. Main conclusions. The above presented analysis of the present state of the topological fixed point property FPP leads to the following inference.

(i) The study of the FPP focuses on three classes of sets: contractible, uniquely arcwise connected (uac), and tree-like continua. Dendroids lie in a center of the classes of sets: they are uac and tree-like, and contractible curves are dendroids. The sets admit natural structures of axiomatic and generalized convexity.

(ii) The most difficult border line of the study of the FPP runs through continua of the lowest dimensions 1, 2 and 3, especially the ones which lie in the usual Euclidean space $E^{3}$. But there are still some important open problems concerning the FPP for sets of an arbitrary dimension - in particular, we can expect a growth of interest in the FPP for products and hyperspaces.

(iii) The topological fixed point property FPP may turn out not only elusive, but even impossible to catch definitively, because it can depend on individual, topologicallyarithmetic properties of continua involved (cf. Hagopian and Mańka 2005, Theorem 3.3). The topologically-arithmetic properties may appear, by nature, quite different from the usual numerical topological invariants as e.g. dimension, degree, etc.

6. The surveys concerning the topological fixed point property after Bing's survey. Let us note that, after the expository article by R. H. Bing 1969, also other surveys and lists of problems concerning the FPP have appeared in the literature. They enlarge the view of the topological fixed point property FPP. We will present them chronologically.

[1] R. H. Bing, The elusive fixed point property, Amer. Math. Monthly 76 (1969), 119-132.

[2] E. Fadell, Recent results in the fixed point theory of continuous maps, Bull. Amer. Math. Soc. 76 (1970), 10-29.

[3] R. F. Brown, On some old problems of fixed point theory, Rocky Mountain J. Math. 4 (1974), 3-14.

[4] R. H. Bing, Commentary on Problem 107, in: The Scottish Book, Birkhäuser, Boston 1981, 190-192.

[5] R. F. Brown, The fixed point property and Cartesian products, Amer. Math. Monthly 89 (1982), 654-678.

[6] Ch. L. Hagopian, Fixed-point problems in continuum theory, in: Continuum Theory and Dynamical Systens (Arcata 1989), Contemp. Math. 117, Amer. Math. Soc., Providence 1991, 79-86.

[7] V. Klee, S. Wagon, Old and New Unsolved Problems in Plane Geometry and Number Theory, Chapter 10: Fixed points, Dolciani Math. Exp., Math. Assoc. America, Washington 1991, 145-150.

[8] Ch. L. Hagopian, Simply connected plane continua have the fixed point property, Topology Proc. 19 (1994), 111-119.

[9] D. P. Bellamy, The fixed point property in dimension one, in: Continua (Cincinnati 1994), Lecture Notes Pure Appl. Math. 170, Dekker, New York 1995, 27-35.

[10] R. F. Brown, Fixed Point Theory, Chapter 10 in: History of Topology, North-Holland, Amsterdam 1999, 271-299. 
[11] K. Kawamura, Fixed Point Theorems, in: Encyclopedia of General Topology, Elsevier, Amsterdam 2004, 402-408.

[12] Ch. L. Hagopian, An update on the elusive fixed-point property, preprint 2006.

Also, we would like to present chronologically the existing surveys on theory of continua because they are naturally concerned, and contain reports on, the topological fixed point property:

[13] W. Lewis, Continuum theory problems, Topology Proc. 8 (1983), 361-394; update: Topology Proc. 9 (1984), 374-382.

[14] M. Barge, M. Brown, Problems in dynamic on continua, in: Continuum Theory and Dynamical Systens (Arcata 1989), Contemp. Math. 117, Amer. Math. Soc., Providence 1991, $177-182$.

[15] A. A. Odintsov, V. V. Fedorchuk, Theory of continua. I, Itogi Nauki i Tekhniki, Algebra. Topology. Geometry, Vol. 29 (in Russian) VINITI, Moscow 1991, 63-119. English transl.: J. Math. Sci. 71 (1994), 2329-2363.

[16] J. C. Mayer, L. G. Oversteegen, Continuum theory, in: Recent Progress in General Topology (Prague 1991), North-Holland, Amsterdam 1992, 453-492.

[17] H. Cook, W. T. Ingram, A. Lelek, A list of problems known as Houston Problem Book, in: Continua (Cincinnati 1994), Lecture Notes Pure Appl. Math. 170, Dekker, New York 1995, 365-398.

[18] V. V. Fedorchuk, A. A. Odintsov, Continuum theory. II. Topology, 1, J. Math. Sci. 74 (1995), 799-812.

[19] J. J. Charatonik, History of continuum theory, in: Handbook of the History of General Topology, Vol. 2, Kluwer Acad. Publ., Dordrecht 1998, 703-786.

[20] J. C. Mayer, L. G. Oversteegen, Continuum theory, in: Encyclopedia of General Topology, Elsevier, Amsterdam 2004, 299-303.

Finally, let us mention the monographic lectures on the FPP at Warsaw University:

[21] K. Sieklucki, Geometric methods of the fixed point theory (in Polish), the academic year 1983/1984 (unpublished notes by Danuta Kołodziejczyk).

[22] K. Sieklucki, Fixed point theory in continua (in Polish), the academic year 1987/1988 (unpublished notes by Marek Gałecki).

\section{Further references}

[23] N. Aronszajn, K. Borsuk, Sur la somme et la produit combinatoire des rétractes absolus, Fund. Math. 18 (1932), 193-197.

[24] W. L. Ayres, Some generalizations of the Scherrer fixed-point theorem, Fund. Math. 16 (1930), 332-336.

[25] H. Bell, On fixed point properties of plane continua, Trans. Amer. Math. Soc. 128 (1967), 539-548.

[26] H. Bell, A fixed point theorem for plane homeomorphisms, Bull. Amer. Math. Soc. 82 (1976), 778-780.

[27] H. Bell, A fixed point theorem for plane homeomorphisms, Fund. Math. 100 (1978), $119-128$. 
[28] H. Bell, Some fixed point theorems, unpublished manuscript 1983, presented in a workshop at the International Congress of Mathematicians, August 16-24, 1983, Warsaw.

[29] D. P. Bellamy, An example of a tree-like continuum without the fixed-point property, in: Proceedings of the International Conference on Geometric Topology (Warsaw 1978), PWN, Warsaw 1980, 35-38.

[30] D. P. Bellamy, A tree-like continuum without the fixed-point property, Houston J. Math. 6 (1980), 1-13.

[31] R. H. Bing, Snake-like continua, Duke Math. J. 18 (1951), 653-663.

[32] R. H. Bing, Challenging conjectures, Amer. Math. Monthly 74 (1967), 56-64.

[33] K. Borsuk, Sur les rétractes, Fund. Math. 17 (1931), 152-170.

[34] K. Borsuk, Einige Sätze über stetige Streckenbilder, Fund. Math. 18 (1932), 198-213.

[35] K. Borsuk, Sur un continu acyclique qui se laisse transformer topologiquement en lui même sans points invariants, Fund. Math. 24 (1935), 51-58.

[36] K. Borsuk, A theorem on fixed points, Bull. Acad. Polon. Sci. Cl. III 2 (1954), 17-20.

[37] K. Borsuk, Theory of Retracts, PWN, Warszawa 1967.

[38] G. E. Bredon, Some examples for the fixed point property, Pacific J. Math. 38 (1971), $571-575$.

[39] L. E. J. Brouwer, On continuous one-to-one transformations of surfaces into themselves, Proc. Kon. Nederl. Akad. Wetensch. Ser. A 11 (1909), 788-798.

[40] L. E. J. Brouwer, Über Abbildung von Mannigfaltigkeiten, Math. Ann. 71 (1912), 97-115.

[41] R. Cauty, Rétractes absolus de voisinage algébriques, Serdica Math. J. 31 (2005), 309-354.

[42] Z. Čerin, J. M. R. Sanjurjo, Universal and proximately universal limits, J. Austral. Math. Soc. Ser. A 61 (1996), 96-105.

[43] H. Cook, Tree-likeness of dendroids and $\lambda$-dendroids, Fund. Math. 68 (1970), 19-22.

[44] J. Dijkstra, An explosion point space without the fixed point property, Topology Appl. 153 (2006), 2948-2951.

[45] J. Dugundji, An extension of Tietze's theorem, Pacific J. Math. 1 (1951), 353-367.

[46] E. Dyer, A fixed point theorem, Proc. Amer. Math. Soc. 7 (1956), 662-672.

[47] C. A. Eberhart, J. B. Fugate, Approximating continua from within, Fund. Math. 72 (1971), 223-231.

[48] E. Fadell, Review of the paper by Bredon 1971, Math. Reviews 46 \# 9962 (1971).

[49] L. Fearnley, D. G. Wright, Geometric realization of a Bellamy continuum, Bull. London Math. Soc. 25 (1993), 177-183.

[50] J. B. Fugate, Retracting fans onto finite fans, Fund. Math. 71 (1971), 113-125.

[51] J. B. Fugate, Small retractions of smooth dendroids onto trees, Fund. Math. 71 (1971), $255-262$.

[52] J. B. Fugate, T. B. McLean, Compact groups of homemorphisms on tree-like continua, Trans. Amer. Math. Soc. 267 (1981), 609-620.

[53] J. B. Fugate, L. Mohler, A note on fixed points in tree-like continua, Topology Proc. 2 (1977), 457-460.

[54] J. B. Fugate, L. Mohler, Fixed point theorems for arc-preserving mappings of uniquely arcwise-connected continua, Proc. Amer. Math. Soc. 123 (1995), 3225-3231.

[55] B. D. Garrett, Almost continuous retracts and the fixed point property for certain nonseparating plane continua, Topology Proc. 28 (2004), 133-142.

[56] R. G. Gurevich, The general point of view on $\lambda$-dendroids, and fixed point theorems (in Russian), Fund. Math. 100 (1978), 109-118. 
[57] A. Gutek, A connected space with a dispersion point without fixed-point property, Topology Appl. 30 (1988), 101-105.

[58] Ch. L. Hagopian, A fixed point theorem for plane continua, Bull. Amer. Math. Soc. 77 (1971), 351-354; Addendum: Bull. Amer. Math. Soc. 78 (1972), 289.

[59] Ch. L. Hagopian, Another fixed point theorem for plane continua, Proc. Amer. Math. Soc. 31 (1972), 627-628.

[60] Ch. L. Hagopian, A fixed point theorem for hyperspaces of $\lambda$ connected continua, Proc. Amer. Math. Soc. 53 (1975), 231-234.

[61] Ch. L. Hagopian, Uniquely arcwise connected plane continua and the fixed-point property, in: Proceedings of the International Conference on Geometric Topology (Warsaw 1978), PWN, Warsaw 1980, 187-188.

[62] Ch. L. Hagopian, Uniquely arcwise connected plane continua have the fixed-point property, Trans. Amer. Math. Soc. 248 (1979), 85-104.

[63] Ch. L. Hagopian, The fixed-point property for deformations of uniquely arcwise connected continua, Topology Appl. 24 (1986), 207-212.

[64] Ch. L. Hagopian, Fixed points of arc-component-preserving maps, Trans. Amer. Math. Soc. 306 (1988), 411-420.

[65] Ch. L. Hagopian, The fixed-point property for simply connected plane continua, Trans. Amer. Math. Soc. 348 (1996), 4525-4548.

[66] Ch. L. Hagopian, The fixed-point property for deformations of tree-like continua, Fund. Math. 155 (1998), 161-176.

[67] Ch. L. Hagopian, Irreducible continua without the fixed-point property, Bull. Polish Acad. Sci. Math. 51 (2003), 121-127.

[68] Ch. L. Hagopian, R. Mańka: Simple spirals on double Warsaw circles, Topology Appl. 128 (2003), 93-101.

[69] Ch. L. Hagopian, R. Mańka: Rational irreducible plane continua without the fixed-point property, Proc. Amer. Math. Soc. 133 (2005), 617-625.

[70] Ch. L. Hagopian, J. R. Prajs, A fixed-point anomaly in the plane, Fund. Math. 186 (2005), 233-249.

[71] O. H. Hamilton, A fixed point theorem for pseudo-arcs and certain other metric continua, Proc. Amer. Math. Soc. 2 (1951), 173-174.

[72] W. Holsztyński, Universal mappings and fixed point theorems, Bull. Acad. Polon. Sci. Sér. Sci. Math. Astronom. Phys. 15 (1967), 433-438.

[73] W. Holsztyński, Fixed points of arcwise connected spaces, Fund. Math. 64 (1969), 289-312.

[74] S. D. Iliadis, Positions of continua on the plane, and fixed points (in Russian), Vestnik Moskov. Univ. Ser. I Mat. Meh., 1970, 66-70.

[75] S. Iliadis, Compact subspaces of $R^{n}$ and fixed points, Serdica 9 (1983), 330-334.

[76] A. Illanes, A tree-like continuum whose cone admits a fixed-point-free map, preprint 2005, to appear in Houston J. Math.

[77] A. Illanes, S. B. Nadler, Jr., Hyperspaces, Monogr. Textbooks Pure Appl. Math. 216, Marcel Dekker, New York 1999.

[78] F. B. Jones, Review of the paper by Mańka 1987a, Zbl. 1988, 0637.54029.

[79] S. Kakutani, Topological properties of the unit sphere of a Hilbert space, Proc. Imp. Acad. Tokyo 19 (1943), 269-271.

[80] J. A. Kennedy, A brief history of indecomposable continua, in: Continua (Cincinnati 1994), Lecture Notes Pure Appl. Math. 170, Dekker, New York 1995, 103-126. 
[81] V. L. Klee, Jr.: Some topological properties of convex sets, Trans. Amer. Math. Soc. 78 (1955), 30-45.

[82] B. Knaster, Un continu dont tout sous-continu est indécomposable, Fund. Math. 3 (1922), 247-286.

[83] B. Knaster, Un théorème sur les fonctions d'ensembles, Ann. Soc. Polon. Math. 6 (1927), 133-134.

[84] B. Knaster, Problem 471 (in French), dated November 3, 1959, The New Scottish Book, deposited in the Library of the Mathematical Institute of Wrocław University; printed in Colloq. Math. 8 (1961), 139.

[85] B. Knaster, Problem 526 (in Polish), dated November 22, 1960, The New Scottish Book, deposited in the Library of the Mathematical Institute of Wrocław University.

[86] B. Knaster, K. Kuratowski, S. Mazurkiewicz, Ein Beweis des Fixpunktsatzes für n-dimensionale Simplexe, Fund. Math. 14 (1929), 132-137.

[87] R. J. Knill, Cones, products and fixed points, Fund. Math. 60 (1967), 35-46.

[88] J. Krasinkiewicz, On the hyperspaces of snake-like and circle-like continua, Fund. Math. 83 (1974), 155-164.

[89] J. Krasinkiewicz, Concerning the boundaries of plane continua and the fixed point property, Bull. Acad. Polon. Sci. Sér. Sci. Math. Astronom. Phys. 21 (1973), 427-431.

[90] K. Kuperberg, Fixed points of orientation reversing homeomorphisms of the plane, Topology Proc. 14 (1989), 195-199.

[91] K. Kuperberg, Fixed points of orientation reversing homeomorphisms of the plane, Proc. Amer. Math. Soc. 112 (1991), 223-229.

[92] A. Lelek, An example of a non-compact locally compact arcwise connected metric space with the fixed point property, Colloq. Math. 24 (1972), 163-168.

[93] W. Lewis, The pseudo-arc, Bol. Soc. Mat. Mexicana (3) 5 (1999), 25-77.

[94] O. V. Lokutsievskiı, A fix-point theorem (in Russian), Uspekhi Mat. Nauk (N.S.) 12 (1957), no. 3 (75), 171-172.

[95] W. Lopez, An example in the fixed point theory of polyhedra, Bull. Amer. Math. Soc. 73 (1967), 922-924.

[96] J. M. Łysko, An example of a contractible continuum without fixed point property for homeomorphisms, Bull. Acad. Polon. Sci. Sér. Sci. Math. Astronom. Phys. 20 (1972), 663-666.

[97] R. Mańka, End continua and fixed points, Bull. Acad. Polon. Sci. Sér. Sci. Math. Astronom. Phys. 23 (1975), 761-766.

[98] R. Mańka, Association and fixed points, Fund. Math. 91 (1976), 105-121; announced in Colloq. Math. 32 (1974), 149.

[99] R. Mańka, On the characterization by Kuratowski of irreducible continua, Bull. Acad. Polon. Sci. Sér. Sci. Math. Astronom. Phys. 26 (1978), 49-55.

[100] R. Mańka, Some problems concerning fixed points, Bull. Acad. Polon Sci. Sér. Sci. Math. Astronom. Phys. 26 (1978), 429-435.

[101] R. Mańka, On uniquely arcwise connected curves, Colloq. Math. 51 (1987), 227-238.

[102] R. Mańka, On fixed point theorems for multifunctions in dendroids, Colloq. Math. 52 (1987), 185-192.

[103] R. Mańka, Connection between set theory and the fixed point property, Colloq. Math. 53 (1987), 177-184.

[104] R. Mańka, On the additivity of the fixed point property for 1-dimensional continua, Fund. Math. 136 (1990), 27-36. 
[105] R. Mańka, On spirals and fixed point property, Fund. Math. 144 (1994), 1-9.

[106] R. Mańka, The fixed point property for some Cartesian products, Fund. Math. 169 (2001), 193-203.

[107] R. Mańka, Cylinders over $\lambda$-dendroids have the fixed point property, Fund. Math. 173 (2002), 101-112.

[108] R. Mańka, General interpretations of Borsuk's fixed point theorem for acyclic curves, preprint 2005.

[109] M. M. Marsh, A fixed point theorem for inverse limits of fans, Proc. Amer. Math. Soc. 91 (1984), 139-142.

[110] M. M. Marsh, A fixed-point theorem for inverse limits of simple n-ods, Topology Appl. 24 (1986), 213-216.

[111] M. M. Marsh, Remark 1, (b) and (c), in the paper by Mańka 1987a, 229-230.

[112] J. Mawhin, Le théorème du point fixe de Brouwer: un siècle de métamorphoses, preprint 2005, to appear in Rev. Histoire Math.

[113] P. Minc, An extension of the Lefschetz fixed theorem to some plane continua, Bull. Acad. Polon. Sci. Sér. Sci. Math. Astronom. Phys. 20 (1972), 871-878.

[114] P. Minc, Generalized retracts and the Lefschetz fixed point theorem, Bull. Acad. Polon. Sci. Sér. Sci. Math. Astronom. Phys. 25 (1977), 291-299.

[115] P. Minc, Local connectedness and fixed points, in: General Topology and its Relations to Modern Analysis and Algebra IV, Part B (Prague 1976), Soc. Czechoslovak Mathematicians and Physicists, Prague 1977, 284-287.

[116] P. Minc, Fixed points and locally connected cyclic continua in $E^{3}$, Fund. Math. 107 (1980), $1-20$.

[117] P. Minc, A fixed point theorem for weakly chainable continua, Trans. Amer. Soc. 317 (1990), 303-312.

[118] P. Minc, A tree-like continuum admitting a fixed point free maps with arbitrarily small trajectories, Topology Appl. 46 (1992), 99-106.

[119] P. Minc, A periodic point free homeomorphism of a tree-like continuum, Trans. Amer. Math. Soc. 348 (1996), 1487-1519.

[120] P. Minc, A weakly chainable tree-like continuum without the fixed point property, Trans. Amer. Math. Soc. 351 (1999), 1109-1121.

[121] P. Minc, A self-map of a tree-like continuum with no invariant indecomposable subcontinuum, Topology Appl. 98 (1999), 235-240.

[122] P. Minc, A hereditarily indecomposable tree-like continuum without the fixed point property, Trans. Amer. Math. Soc. 352 (2000), 643-654.

[123] L. Mohler, The fixed point property for homeomorphisms of 1-arcwise connected continua, Proc. Amer. Math. Soc. 52 (1975), 451-456.

[124] S. B. Nadler, Jr., Hyperspaces of Sets, Monogr. Textbooks Pure Appl. Math. 49, Marcel Dekker, New York 1978.

[125] P. Niemiec, Locally arcwise connected metrizable spaces with the fixed point property are complete-metrizable, Topology Appl. 153 (2006), 1639-1642.

[126] G. Nöbeling, Eine Fixpunkteigenschaft der Baumkurven, Ergebnisse eines math. Kolloquium 2 (1932), 19.

[127] V. P. Okhezin, Fixed-point theorems on products of spaces (in Russian), in: Studies in Functional Analysis and its Applications, Ural. Gos. Univ., Sverdlovsk 1985, 72-80.

[128] V. P. Okhezin, On the fixed-point theory for non-compact maps and spaces. I, Topol. Methods Nonlinear Anal. 5 (1995), 83-100. 
[129] L. G. Oversteegen, J. T. Rogers, Jr., An inverse limit description of an atriodic tree-like continuum and an induced map without a fixed point, Houston J. Math. 6 (1980), 549-564.

[130] L. G. Oversteegen, J. T. Rogers, Jr., Fixed-point-free maps on tree-like continua, Topology Appl. 13 (1982), 85-95.

[131] L. G. Oversteegen, E. D. Tymchatyn, On atriodic tree-like continua, Proc. Amer. Math. Soc. 83 (1981), 201-204.

[132] P. L. Papini, Nonexpansive maps and fixed points, in: Handbook of Convex Geometry, Vol. A, B, North-Holland, Amsterdam 1993, 1221-1236.

[133] J. M. R. Sanjurjo, Stability of the fixed point property and universal maps, Proc. Amer. Math. Soc. 105 (1989), 221-230.

[134] J. P. Schauder, Der Fixpunktsatz in Funktionalräumen, Studia Math. 2 (1930), 171-180.

[135] W. Scherrer, Über ungeschlossene stetige Kurven, Math. Z. 24 (1926), 125-130.

[136] Yu. A. Shashkin, Nepodvizhnye tochki (in Russian), Nauka, Moscow 1989, English transl. by Victor Minakhin: Fixed Points, Amer. Math. Soc., Providence; Math. Assoc. America, Washington 1991.

[137] K. Sieklucki, A theorem on fixed points for some plane acyclic continua, Bull. Acad. Polon. Sci. Sér. Sci. Math. Astronom. Phys. 15 (1967), 377-379.

[138] K. Sieklucki, On a class of plane acyclic continua with the fixed point property, Fund. Math. 63 (1968), 257-278.

[139] K. Sieklucki, Generalized retractions and fixed points, Bull. Acad. Polon. Sci. Sér. Sci. Math. Astronom. Phys. 19 (1971), 945-949.

[140] K. Sieklucki, A fixed point problem and the Hilbert cube, Bull. Acad. Polon. Sci. Sér. Sci. Math. Astronom. Phys. 22 (1974), 421-426.

[141] K. Sieklucki, Shift-invariant subsets of the Hilbert cube, Bull. Acad. Polon. Sci. Sér. Sci. Math. Astronom. Phys. 23 (1975), 1265-1272.

[142] K. Sieklucki, The fixed point property for 1-dimensional continua, in: Proceedings of the International Conference on Geometric Topology (Warsaw, 1978), PWN, Warsaw 1980, 409-410.

[143] K. Sieklucki, Generalized cones and the fixed point property, Bull. Polish Acad. Sci. Math. 33 (1985), 173-176.

[144] M. Sobolewski, A uniquely arcwise connected continuum without the fixed point property, Bull. Polish Acad. Sci. Math. 34 (1986), 307-313.

[145] M. Sobolewski, A stronger fixed point property, Preliminary report in the topological seminar at Warsaw University.

[146] M. Sobolewski, A weakly chainable uniquely arcwise connected continuum without the fixed point property, preprint presented at Special Session of Joint AMS-SMM International Meeting Celebrates Prof. Bellamy 60th Birthday, Houston, TX, USA, May 13-15, 2004.

[147] M. Sobolewski, A curve with the fixed point property whose cylinder admits a fixed point free map, Houston J. Math. 31 (2005), 239-253.

[148] M. Shtanko, Continua with the fixed-point property (in Russian), Dokl. Akad. Nauk SSSR 154 (1964), 1291-1293.

[149] A. Tarski, A fixpoint theorem for lattices and its applications (preliminary report), Bull. Amer. Math. Soc. 55 (1949), 1051-1052 and 1192.

[150] A. Tarski, A lattice-theoretical fixpoint theorem and its applications, Pacific J. Math. 5 (1955), 285-309.

[151] R. B. Thompson, Weak semicomplexes and the fixed point theory of tree-like continua, Duke Math. J. 38 (1971), 211-219. 
[152] A. Tominaga, The fixed point property for continua approximated from within by Peano continua with this property, Proc. Amer. Math. Soc. 91 (1984), 444-448.

[153] A. Tominaga, The fixed point property for the cone over the Cartesian product of chainable continua, Bull. Polish Acad. Sci. Math. 35 (1987), 337-339.

[154] A. Tominaga, A note on a fixed point theorem of Okhezin, Proc. Amer. Math. Soc. 114 (1992), 1139-1143.

[155] A. Tychonoff, Ein Fixpunktsatz, Math. Ann. 111 (1935), 767-776.

[156] I. Verchenko, Sur les continus acycliques transformés en eux-même d'une manière continue sans points invariants (in Russian), Mat. Sbornik (N.S.) 8 (50) (1940), 295-306.

[157] T. van der Walt, Fixed and almost fixed points, Mathematical Centre Tracts 1, Mathematisch Centrum, Amsterdam 1963.

[158] L. E. Ward, Jr., Characterization of the fixed point property for a class of set-valued mappings, Fund. Math. 50 (1961/1962), 159-164.

[159] L. E. Ward, Jr., A general fixed point theorem, Colloq. Math. 15 (1966), 243-251.

[160] G. S. Young, The introduction of local connectivity by change of topology, Amer. J. Math. 68 (1946), 479-494.

[161] G. S. Young, Fixed point theorems for arcwise connected continua, Proc. Amer. Math. Soc. 11 (1960), 880-884.

[162] W. L. Young, A product space with the fixed point property, Proc. Amer. Math. Soc. 25 (1970), 313-317. 